\title{
P04-2-21 Poster session
}

\section{The mechanism underlying the modulatory action of amniodarone on hERG K+ channels}

\section{Chung-Chin Kuo ${ }^{1,2}$, Yung-Chen Lo ${ }^{1}$}

\author{
${ }^{I}$ Department of Physiology, National Taiwan University College of Medicine, Taiwan, ${ }^{2}$ Department of Neurology, \\ National Taiwan University Hospital, Taiwan
}

Background: hERG K+ channels could manifestly modulate the duration of an action potential, and also the occurrence of the subsequent one if there are repetitive discharges. The effect is achieved by the strong voltage-dependent (depolarization-driven) activation and rapid inactivation, and also the large tail current upon repolarization of hERG K+ channels. Amiodarone, a widely prescribed type III antiarrhythmics, is well known for its inhibitory effect on hERG K+ channels, and is also renowned for its extraordinarily low risk of causing tachyarrythmia such as Torsade de Pointes, the mechanism underlying which has not been fully resolved.

Methods: With heterologous expression of hERG K+ channels in Xenopus oocytes, we recorded the currents and studied the effect of amniodarone with two-electorde whole-cell voltage-clamp techniques.

Results: 10 micromolar amiodarone inhibits the outward hERG K+ currents elicited by a pulse of moderate depolarization with apparent acceleration of the macroscopic inactivation. The inward tail hERG $\mathrm{K}+$ currents upon the following repolarization could also be inhibited, although the extent of inhibition would be significantly different according to the length of the depolarization and the voltage of the repolarization. 10 micromolar amiodarone, on the other hand, could enhance hERG currents elicited by a mild depolarization pulse, especially if following a positive prepulse separated by a gap. The enhancement effect is dependent on the voltage and duration of both the prepulse and the gap.

Conclusions: Amiodarone acts chiefly as a gating modifier rather than a pore blocker of the hERG K+ channel, modifying not only activation and inactivation, but also recovery from inactivation. Also, the modulatory effect of amiodarone probably could be more appropriately envisaged with the changes in the key transitional kinetics than just the steady-state distribution of different gating states. The apparent use-dependent inhibitory and reverse use-dependent enhancement effect of amiodarone on hERG K+ currents may explain its effectiveness in not only lengthening the action potential but also offsetting the driving forces for early afterdepolarization and consequent abnormal cardiac rhythms. 